\section{Nanotubes in Microwave Fields: Light Emission, Intense Heat, Outgassing, and Reconstruction}

T. J . Imholt, ${ }^{\dagger}$ C. A. Dyke, ${ }^{\ddagger}$ B. Hasslacher, ${ }^{\S}$ J. M. Perez,,$+\uparrow$ D. W. Price, ${ }^{\ddagger}$ J. A. Roberts,,,+ J. B. Scott, ${ }^{\dagger}$ A. Wadhawan, ${ }^{\dagger}$ Z. Y $e^{\dagger}{ }^{\dagger}$ and J. M. Tour*,

Department of Physics, University of North Texas, 211 Ave. A, Denton, Texas 76201, Departments of Chemistry and Mechanical Engineering and

Materials Science, and Center for Nanoscale Science and Technology, MS 222, Rice University, 6100 Main Street, Houston, Texas 77005, and Institute for Physical Sciences, P.O. Box 3541, Santa Fe, New Mexico 87501

Received J une 24, 2003

Revised Manuscript Received September 9, 2003

Single-walled carbon nanotubes (SWNTs) exhibit diverse and unique properties. Recently, a surprising feature has been the ignition of nanotubes in the presence of an ordinary camera flash. ${ }^{1} \mathrm{Here}$, we report that SWNTs, produced via the HiPco process, ${ }^{2}$ display strong microwave absorption $\left(1.01 \times 10^{-5} \mathrm{eV}\right.$ microwave field) with subsequent dramatic light emission, intense heat release, outgassing, and nanotube reconstruction.

The microwave source used for this experiment was a 700-W magnetron operating at $2.45 \mathrm{GHz}$. SWNTs were tested in both purified ${ }^{3}$ and raw conditions. All visual reactions took place $\sim 1 \mathrm{~s}$ after application of the microwave field. Laser-oven-produced SWNTs were also tested, but the effects were far less dramatic. This might be due to increased average diameters of the SWNTs in the laser-oven materials or differing percentages of (a) metallic to semiconducting tubes, (b) iron seed particles at the ends of the tubes, or (c) defects in the tubes.

In air and under application of the microwave field, unpurified HiPco SWNTs ignited and burned. ${ }^{4}$ The regions of the SWNTs that underwent this process showed a permanent color change to orange. These orange regions fluoresce under normal room light. A TEM image of these orange regions shows a change to amorphous carbon structures that are $50-500 \mathrm{~nm}$ in diameter with little discerni ble tube structures. Powder $\mathrm{X}$-ray diffraction analysis on the orange material confirmed the presence of hematite. The purified nano-

* Towhomcorrespondenceshould beaddressed. E-mail: tour@rice.edu; roberts@unt.edu; jperez@unt.edu.

+ University of North Texas.

‡ Rice University.

$\S$ Institute for Physical Sciences.

(1) Ajayan, P. M.; Terrones, M.; de la Guardia, A.; Huc, V.; Grobert N.; Wei, B. Q.; Lezec, H.; Ramanath, G.; Ebbesen, T. W. Science 2002, 296, 705.

(2) Nikolaev, P.; Bronikowski, M. J .; Bradley, R. K.; Rohmund, F.; Colbert, D. T.; Smith, K. A.; Smalley, R. E. Chem. Phys. Lett. 1999, 313, 91.

(3) Chiang, I. W.; Brinson, B. E.; Huang, A. Y.; Willis, P. A.; Bronikowski, M. J .; Margrave, J . L.; Smalley, R. E.; Hauge, R. H. J Phys. Chem. B. 2001, 105, 8297.

(4) Interestingly, we observed that a magnifying glass and sunlight can also cause the raw HiPco tubes to rapidly ignite and burn.

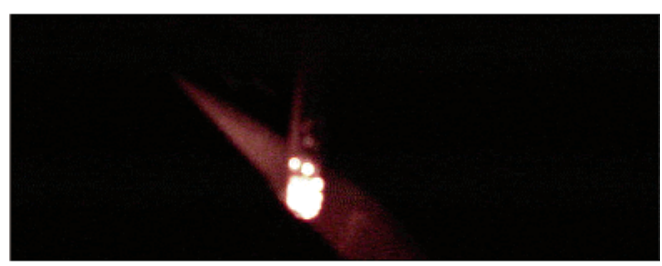

Figure 1. SWNTs in a UHV tube suspended above $(\sim 8 \mathrm{~cm})$ an active microwave source show the white light emission. Room lights were off.

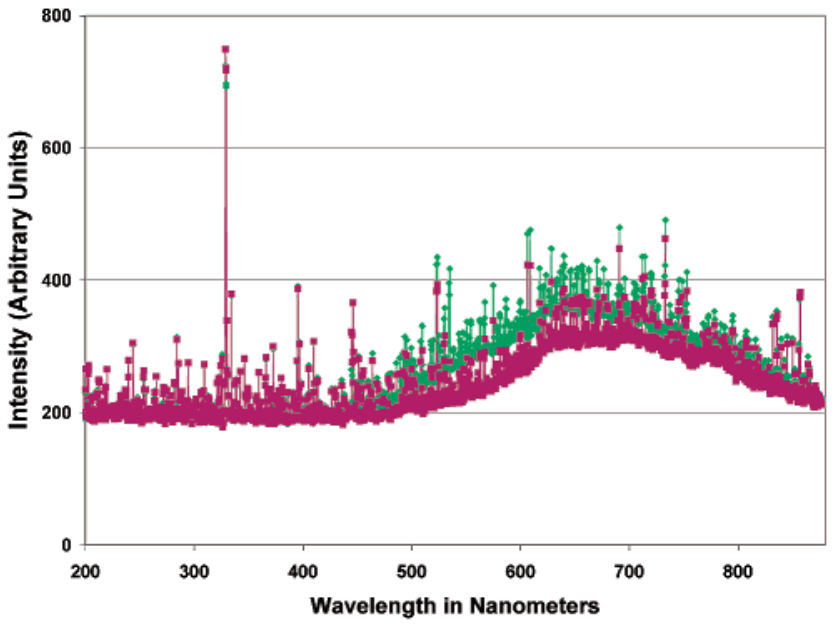

Figure 2. Spectrum of light from raw (green) and purified (burgundy) SWNTs when subjected to the microwave irradiation. The background spectrum showed no peaks.

tubes, when in the presence of the microwave field in air, only display random scintillations of white light.

Both the raw and purified nanotubes, when placed under UHV in the presence of the microwave field, emitted white light (Figure 1). The process could be repeated with no observable degradation of the light intensity over $\sim 20 \mathrm{~s}$ of microwave irradiation. After minutes of constant exposure, there was a diminution of light, though they were still light-emitting. Short exposure pulses $(\sim 3-5 \mathrm{~s})$ could be repeated with no obvious degradation over the 35 pulses attempted.

The light emission spectra in this experiment were taken with a fiber optic spectrometer with collimating lens for increased sensitivity. These emissions extend from the IR through visible and into the UV regions of the electromagnetic spectrum (F igure 2). The majority of the broadband emissions are in the visible and NIR regions with the UV components consisting mainly of spikes corresponding to known atomic emissions assigned to carbon $(\sim 330 \mathrm{~nm})$, iron (the catalyst used in the HiPco process), or hydrogen (Figure 2). Although the light emission could be from a frequency up-conversion, it is more likely due to a broadband photon emission from the hot tubes (vide infra). Light emissions from carbon nanostructures, such as $C_{60}$, have been observed. ${ }^{5}$

The light emission under UHV conditions is accompanied by outgassing in both the crude and purified

(5) Palstra, T. T. M.; Haddon, R. C.; Lyons, K. B. Carbon 1997, 35, 1825 


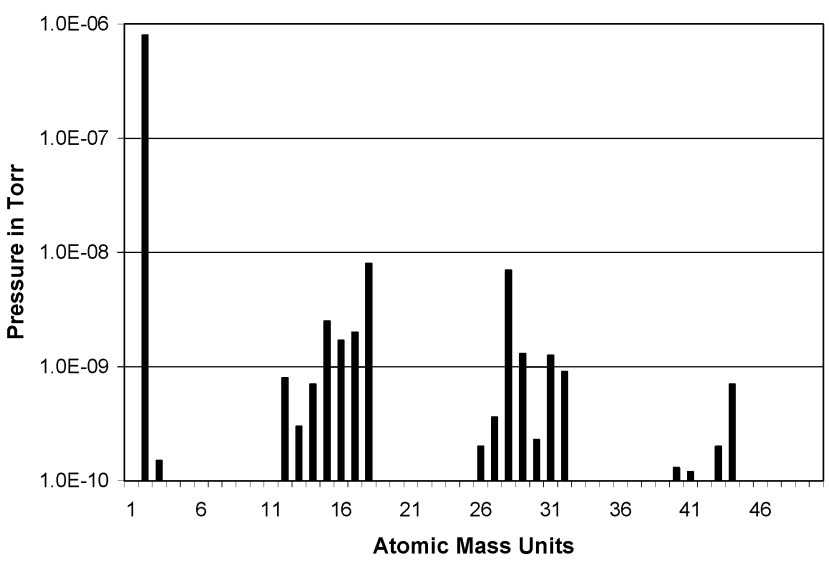

Figure 3. RGA (Dycor LC200). Shown are the masses (amu) of the species evolved from the UHV tube upon microwave irradiation with a large initial peak from $\mathrm{H}_{2}(2 \mathrm{amu})$. Trace background constituents of the vacuum system (recorded prior to opening the nanotube sample to the vacuum system) were subtracted out of this spectrum.

tubes. The expelled gas, seen after several seconds of application of the microwave field, was observed in a residual gas analyzer (RGA) (Figure 3). Nanotubes are known to absorb hydrogen; ${ }^{6}$ therefore, its presence may be from small amounts of adventitious hydrogen present in the laboratory environment or as a pyrolysate from absorbed organics such as solvent or pump oil. But there is clearly nanotube breakdown at the higher temperatures and prolonged (seconds) exposure times. Use of lower power microwaves are now being studied for hydrogen outgassing without concomitant nanotube breakdown.

In addition to the light emission and outgassing, the microwave irradiation of the samples was accompanied by a rapid temperature increase in the sample. ${ }^{7}$ Pyrometer measurements showed temperatures reaching 2000 ${ }^{\circ} \mathrm{C}$, and upon removal of the microwave field a thermocouple was immediately attached to the quartz vessel and a temperature of $1550{ }^{\circ} \mathrm{C}$ was observed.

The heat release, light emission, and gas evolution were further accompanied by the nanotube samples undergoing intense mechanical motion. The initial nanotube material spreads to about twice its original volume when the microwave field is applied, and when the microwave field is turned off, the sample contracts back to its near-original size. This occurs in a repeatable manner upon the short exposure cycles. Repeated exposure caused a decrease in the observed mechanical motion presumably from cross-linking or welding of the tubes. $^{8}$ Indeed, TEM imaging of the nanotubes after

(6) (a) Pradhan, B. K.; Harutyunyan, A. R.; Stojkovic, D.; Grossman, J . C.; Zhang, P.; Cole, M. W.; Crespi, V.; Goto, H.; Fuliwara, J .; Eklund, P. C. J . Mater. Res. 2002, 17, 2209. (b) Ma, Y.; Xia, Y.; Zhao, M.; Ying, M. Phys. Rev. B 2002, 65, 155430. (c) Gundiah, G.; Govindaraj, A. Rajalakshmi, N.; Dhathathreyan, K. S.; Rao, C. N. R. J . Mater. Chem. 2003, 13, 209. (d) Gordillo, M. C.; Boronat, J .; Casulleras, J. Phys. Rev. Lett. 2000, 85, 2348.

(7) The temperatures here exceed those commonly observed by microwave superheating. See: Baghurst, D. R.; Mingos, D. M. P. J . Chem. Soc., Chem. Commun. 1992, 6, 674.

(8) (a) Terrones, M.; Banhart, F.; Grobert, N.; Charlier, J --C.; Terrones, H.; Ajayan, P. M. Phys. Rev. Lett. 2002, 89, 075505. (b) Tsai S. H.; Shiu, C. T.; J ung, W. J .; Shih, H. C. Carbon 2000, 38, 1879. (c) Baughman, R. H. Science 2002, 297, 787. (d) Zhao, Y.; Yakobson, B. I.; Smalley, R. E. Phys. Rev. Lett. 2002, 88, 185501. (e) Zhao, Y.; Smalley, R. E.; Yakobson, B. I. Phys. Rev. B 2002, 66, 195409.

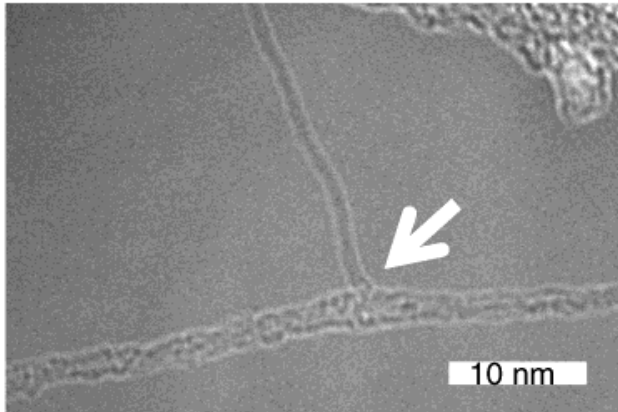

Figure 4. TEM image of the fused nanotubes after microwave irradiation. There were numerous such regions observed in the irradiated samples. A portion of the lacey carbon grid is evident in the top right portion of the photo.

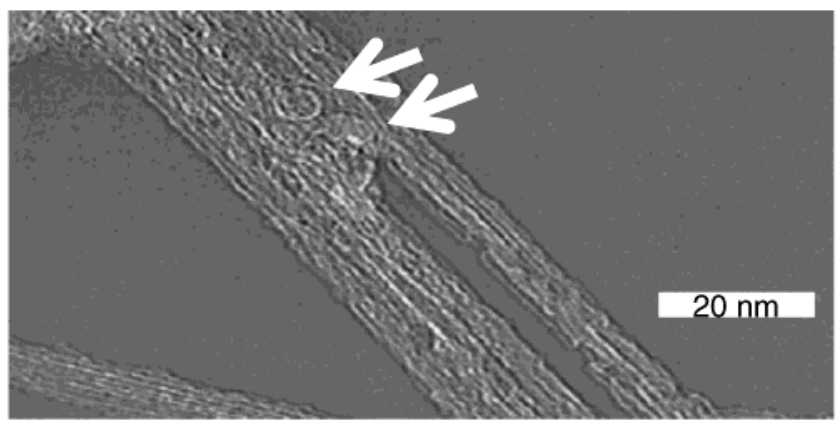

Figure 5. TEM image showing looped nanotubes after microwave irradiation. There were numerous such regions observed in the irradiated samples.

extended microwave irradiation in UHV showed that many of the nanotubes fused (welded) into neighboring tubes to form junctions. The well-defined junction formations can be seen in Figure 4 and it is very similar in configuration to theoretical models. ${ }^{8 d}$ This might prove to be an efficient means of welding nanotubes or nanotube-based ropes $^{9}$ after dispersion in blends or composites, 10 thereby locally increasing the modulus of the microwave-exposed regions. Additionally, formations of looped structures were abundant in the irradiated tubes (Figure 5). The welding of SWNTs requires breaking of carbon-carbon bonds and rearrangement of the carbon atoms. For this to take place, temperatures must reach at least $1500^{\circ} \mathrm{C},{ }^{1}$ indicative of an efficient absorption of microwaves. Neither welds nor loops are present in the original HiPco SWNTs. 2,3

In summary, HiPco-generated SWNTs display strong microwave absorption with subsequent dramatic light emission, intense heat release, outgassing, and nanotube reconstruction.

Acknowledgment. U. North Texas acknowledges support from the NSF DMR-0074636 and the Texas Advanced Technology Program under Award No. 0035940048-1999. Rice University acknowledges support from NASA-J SC-NCC-9-77, NCC-01-0203, NSF-DMR0073046, and AFOSR-F 49620-01-1-0364. T.J .I. thanks D. Allara. J .M.T. thanks W. Guo for the TEM analysis.

\section{034530G}

(9) J iang, K.; Li, Q.; Fan, S. Nature 2002, 419, 801.

(10) Mitchell, C. A.; Bahr, J. L.; Arepalli, S.; Tour, J. M.; Krishnamoorti, R. Macromol ecules 2002, 35, 8825. 